\title{
Underwater Passive Acoustic localization of Pacific Walruses in the NORTHEASTERN CHUKCHI SEA
}

\author{
Brendan Rideout ${ }^{1,2}$, Stan E. Dosso ${ }^{1}$, and David Hannay ${ }^{2}$ \\ ${ }^{1}$ School of Earth and Ocean Sciences, University of Victoria, 3800 Finnerty Rd., Victoria, BC, Canada, V8P 5C2, \\ bprideou@hawaii.ca \\ ${ }^{2}$ JASCO Research Ltd., 2305-4464 Markham St., Victoria, BC, Canada, V8Z 7X8
}

\section{INTRODUCTION}

This paper presents results from an MSc thesis [1] research project whose aim is the passive acoustic localization, and localization uncertainty analysis, of vocalizing, submerged Pacific walruses (Odobenus rosmarus divergens) when the acoustic propagation environment and receiver locations are not precisely known. In this case, passive acoustic localization involves using the natural vocalizations of the walrus to estimate the three-dimensional (3-D) position of the animal. The primary goal of this work is to provide a tool for collecting biological data (e.g., underwater swim speed) on wild walruses and other vocalizing underwater marine mammals which would not be possible with twodimensional methods and which provides a rigorous uncertainty estimate.

To perform localization, arrival times for direct and interface-reflected (i.e., sound which reflects off the surface and/or bottom) acoustic paths at a set of underwater acoustic receivers are processed using an iterated linearized Bayesian inversion of the ray-tracing equations. Environmental parameters (water depth and sound speed correction) and receiver parameters (3-D hydrophone locations and synchronization times), in addition to walrus positions and vocalization times, are treated as unknowns and constrained with prior estimates and prior uncertainties. Prior estimates for walrus positions and times are given large uncertainties so as not to significantly influence the final solution. Maximum a posteriori (MAP) estimates for all unknowns (walrus positions and vocalization times, environmental parameters, and receiver parameters) are calculated simultaneously. To treat cases where both the data errors (i.e., arrival time) and prior uncertainties are only known in a relative sense, an inversion approach previously developed for array element localization [2] is extended here using the Akaike Bayesian information criterion (ABIC) [3] (a maximum entropy condition).

\section{THEORY}

Let $\mathbf{m}$ be the vector of unknown model parameters (i.e., 3-D locations and times for each vocalization, environmental parameters, and receiver parameters) and $\mathbf{t}$ be the vector of direct and interface-reflected arrival times for each of the vocalizations. The relationship between $\mathbf{m}$ and $\mathbf{t}$ is nonlinear, but can be linearized about an arbitrary starting model $\mathbf{m}_{\mathbf{0}}$ by retaining the first order term from the Taylor expansion:

$$
\mathbf{t}=\mathbf{t}(\mathbf{m})=\mathbf{t}\left(\mathbf{m}_{0}+\delta \mathbf{m}\right) \approx \mathbf{t}\left(\mathbf{m}_{0}\right)+\mathbf{J} \delta \mathbf{m} .
$$

In this case, $\mathbf{J}$ is the Jacobian matrix composed of partial derivatives evaluated at $\mathbf{m}_{0}$. Rearranging (1) and taking $\mathbf{m}-$ $\mathbf{m}_{\mathbf{0}}=\delta \mathbf{m}$ yields:

$\mathbf{d} \equiv \mathbf{t}-\mathbf{t}\left(\mathbf{m}_{0}\right)+\mathbf{J} \mathbf{m}_{\mathbf{0}}=\mathbf{J} \mathbf{m}$.

This yields an inverse problem which is ill-conditioned due to both the source and receiver positions being unknown. Here, regularization is used to stabilize the problem and incorporate prior information (e.g., measurements of receiver positions and water depth) into the problem. As a result, in seeking to estimate the most probable walrus location, the following objective function is minimized over m:

$$
\begin{aligned}
& \Theta(\mathbf{m})=[\mathbf{d}-\mathbf{d}(\mathbf{m})]^{T} \mathbf{C}_{\mathbf{d}}^{\prime-1}[\mathbf{d}-\mathbf{d}(\mathbf{m})] \\
& +\mu[\mathbf{m}-\hat{\mathbf{m}}]^{T} \mathbf{C}_{\hat{\mathbf{m}}}^{\prime-1}[\mathbf{m}-\hat{\mathbf{m}}]
\end{aligned}
$$

where $\mathbf{C}_{\mathbf{d}}^{\prime}$ and $\mathbf{C}_{\hat{\mathbf{m}}}^{\prime}$ are the relative data covariance and relative prior covariance matrices, respectively, $\hat{\mathbf{m}}$ is the vector of prior estimates, and $\mathbf{d}(\mathbf{m})$ is the model-predicted data vector. It can be shown that the MAP solution for $\mathbf{m}$ is:

$\mathbf{m}_{\mathrm{MAP}}=\hat{\mathbf{m}}+\left[\mathbf{J}^{T} \mathbf{C}_{\mathbf{d}}^{\prime-1} \mathbf{J}+\mu \mathbf{C}_{\hat{\mathbf{m}}}^{\prime-1}\right]^{-1} \mathbf{J}^{T} \mathbf{C}_{\mathbf{d}}^{-1}[\mathbf{d}-\mathbf{J} \hat{\mathbf{m}}]$.

The optimum value of the trade-off parameter $\mu$ is selected by minimizing the ABIC over $\mu$. The derivation and expression for the ABIC can be found in Ref. [1]. After calculating $\mathbf{m}_{\mathrm{MAP}}$ for this iteration, $\mathbf{m}_{\mathrm{MAP}}$ is set as the starting model for the next iteration; due to the linearization step, the solution converges over several iterations. Following convergence, the posterior covariance matrix is calculated:

$\mathbf{C}_{\mathbf{m}}=\sigma_{0}^{2}\left[\mathbf{J}^{T} \mathbf{C}_{\mathbf{d}}^{\prime-1} \mathbf{J}+\mu \mathbf{C}_{\hat{\mathbf{m}}}^{\prime-1}\right]^{-1}$

where $\sigma_{0}{ }^{2}=\frac{\Theta\left(\mathbf{m}_{\mathrm{MAP}}\right)}{N}$ and $N$ is the number of data. This matrix includes parameter variances as diagonal elements and covariances as off-diagonal elements. 
In some instances, knowing the relative localization uncertainty between estimated source positions (e.g., when estimating the uncertainty in a swim speed measurement) may be more informative than the absolute uncertainty values given by diagonal elements of $\mathbf{C}_{\mathbf{m}}$. It can be shown that the relative uncertainty between two estimated model parameters is:

$\sigma_{p q}=\left[C_{m, p, p}+C_{m, q, q}-2 C_{m, p, q}\right]^{1 / 2}$

where $C_{m, p, p}$ is the posterior variance of $\mathrm{m}_{\mathrm{p}}$ and $C_{m, p, q}$ is the covariance of $m_{p}$ and $m_{q}$.

\section{RESULTS AND DISCUSSION}

From August to October 2009, a set of 40 underwater acoustic recorders were deployed throughout the northeastern Chukchi Sea, northwest of Alaska, to record marine mammal vocalizations. These recorders continuously collected 24-bit acoustic data with a sampling frequency of $16 \mathrm{kHz}$. Three of these recorders were deployed approximately $400 \mathrm{~m}$ apart near the Hanna Shoal, a $\sim 30 \mathrm{~m}$ deep region of the Chukchi Sea where Pacific walruses are known to congregate during the summer months. Given that water depth was essentially uniform, a range independent propagation environment is assumed.

Pacific walruses are considered a gregarious animal, and make a variety of different vocalizations both in-air and underwater. One of the underwater vocalizations, called a 'knock', is particularly well suited for localization due to its impulsive-like characteristics (i.e., short duration and fast rise time). From the Hanna Shoal data, a sequence of 11 walrus knocks was localized using the approach outlined in the previous section. The results of this localization are shown in Figure 1. To perform this localization, arrival times for the direct and interface-reflected rays at each of the three hydrophones were identified. The error bars in Figure 1 represent single standard deviations. The slope of the least-squares linear regression fit to the estimated walrus position and vocalization time values yields an estimated walrus swim speed of $(0.98 \pm 0.19) \mathrm{m} / \mathrm{s}$, with swim speed uncertainty estimated using a Monte Carlo approach.

Given the relative and absolute uncertainties, several observations can be made about the estimated track. The zig-zags in the track in the $x-y$ plane are within relative uncertainties and, thus, are unlikely to be real. Also, there is limited evidence that the walrus is deeper at the end of the track than at the beginning.

The uses of this technique are not limited to locating Pacific walruses; other marine mammals (such as sperm whales) which produce impulsive-like vocalizations, and nonbiologic sources, could potentially be located.
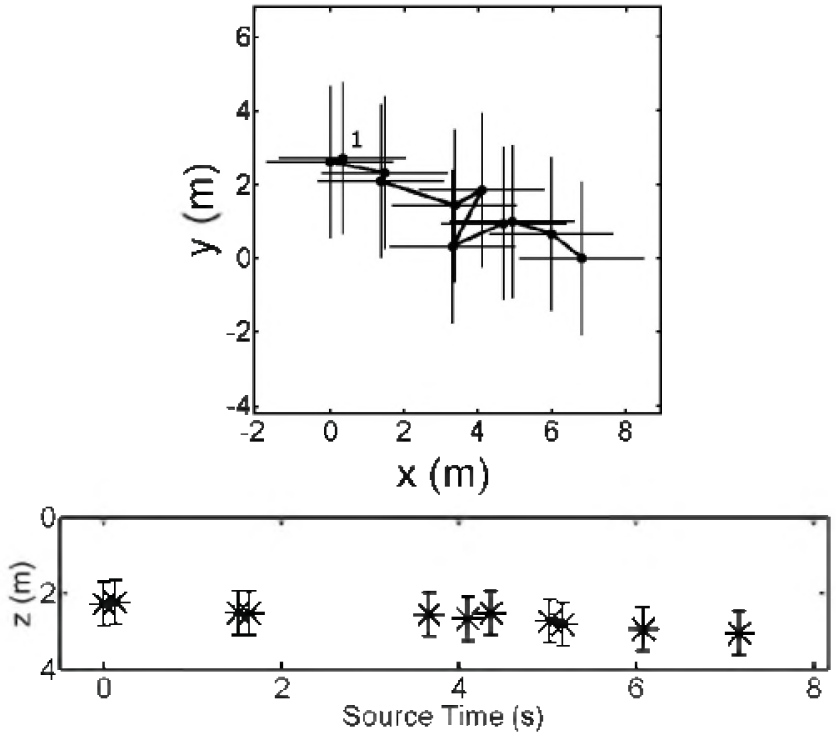

Figure 1 - Estimated horizontal position with relative $x$ and $y$ uncertainties (top) and estimated $z$ position with absolute uncertainties for each of the eleven walrus knocks. The earliest knock in the top plot is labeled. Straight lines between knock locations are meant to illustrate the order in which the knocks were produced, and do not necessarily indicate the path the walrus took.

\section{REFERENCES}

[1] Rideout, B. P. (2011). Underwater Acoustic Localization and Tracking of Pacific Walruses in the Northeastern Chukchi Sea. MSc. Thesis, University of Victoria.

[2] Dosso, S. E. et al. (1998). Array element localization for horizontal arrays via Occams inversion. J. Acoust. Soc. Am., 104, 846-859.

[3] Akaike, H. (1974). A new look at the statistical model identification. IEEE Trans. Auto. Cont., 19, 716-723.

\section{ACKNOWLEDGEMENTS}

The authors would like to thank JASCO Research Ltd., ConocoPhillips Company, and Shell Exploration and Production Company for allowing access to underwater acoustic data collected on their behalf during the summer of 2009. This work was supported in part by the Natural Sciences and Engineering Research Council, the British Columbia Ministry for Advanced Education, and the National Science Foundation.

\section{AUTHOR NOTES}

The work was conducted while Brendan Rideout was a student at the University of Victoria in Victoria, British Columbia. He is currently a $\mathrm{PhD}$ student at the University of Hawaii. 